\title{
STAR Vertex Detector Upgrade Development
}

\author{
L. C. Greiner ${ }^{1}$, H. S. Matis, T. Stezelberger, C. Q. Vu, H. Wieman
}

Lawrence Berkeley National Laboratory

1 Cyclotron Road

MS 70R0319,

Berkeley, CA, USA

E-mail: lcgreiner@1bl.gov

\section{Szelezniak}

Institut Pluridisciplinaire Hubert Curien,

23 rue du Loess, 67037 Strasbourg, France

\section{Sun}

Institute of High Energy Physics

Beijing 100039, China

\begin{abstract}
We report on the development and prototyping efforts undertaken with the goal of producing a micro-vertex detector for the STAR experiment at the RHIC accelerator at BNL. We present the basic detector requirements and show a sensor development path, conceptual mechanical design candidates and readout architecture. Prototyping and beam test results with current generation MimoSTAR-2 sensors and a readout system featuring FPGA based on-the-fly hit finding and data sparsification are also presented.
\end{abstract}

The 16th International Workshop on Vertex detectors

Lake Placid, NY, USA

23-28 September, 2007

\footnotetext{
$1 \quad$ Speaker
} 


\section{Introduction}

The STAR Heavy Flavor Tracker (HFT) upgrade group is working to extend the capabilities of the STAR detector [1] in the heavy flavor domain by providing a tracking system that will allow for high resolution vertex measurements. This upgrade is designed to allow for the direct topological reconstruction of $\mathrm{D}$ and $\mathrm{B}$ mesons through the identification of decay vertices displaced from the primary interaction vertex by $100-150 \mu \mathrm{m}$ [2]. The Pixel detector is composed of two layers of high resolution Monolithic Active Pixel Sensors (MAPS) and is the highest resolution and innermost detector of the vertex detector upgrades for STAR.

Achieving the required sub $40 \mu \mathrm{m}$ extrapolated track impact parameter resolution in the Pixel detector is quite challenging in many ways. Multiple coulomb scattering constrains the detector to a design limit of $<0.5 \%$ radiation length per layer in the tracking region. This small material budget needs to contain the MAPS sensors, a readout cable and a highly rigid support structure to maintain the position of the pixels, within an internal detector reference system, to $10 \mu \mathrm{m}$. In this stringent environment, the mechanical design requirements interact strongly with the sensor and readout electronics design. Sensors thinned to $50 \mu \mathrm{m}$, air cooling, a $500 \mu \mathrm{m}$ thick beryllium beam pipe, and aluminum rather than copper conductor readout cables become necessary aspects of the design. The expected final system design is an array of 33 sensor ladders with ten $2 \mathrm{~cm} \times 2 \mathrm{~cm}$ sensors per ladder and parallel independent ladder readout systems. Each sensor is a $640 \times 640$ array of $30 \mu \mathrm{m}$ square pixels giving the Pixel detector system a total pixel count of more than $135 \mathrm{M}$. The angular coverage of the Pixel detector $(|\eta| \leq$ 1) is matched to that of the outer tracking detectors. The final extrapolated track impact parameter resolution is achieved by using the existing tracking detectors of the STAR experiment with the addition of a two layer silicon strip detector just outside of the Pixel detector. Tracking is performed from the outside of the STAR experiment inward to the vertex with increasingly higher resolution until the final resolution of $30 \mu \mathrm{m}$ is achieved with the addition of the high resolution Pixel track points. We are pursuing an incremental approach to reaching the final design by constructing prototype generations of sensors and readout electronics.

\section{Sensor Development Path}

We are working in collaboration with the CMOS and ILC group at the Institut Pluridisciplinaire Hubert Curien (IPHC) in Strasbourg, France to produce sensors that meet the requirements of the STAR Pixel detector. Our sensor development path for the Pixel detector sensors is tailored to follow the development path of the technology as set by the IPHC group. In this path, MAPS sensors [3] with multiplexed serial analog outputs in a rolling shutter configuration are the first generation of sensor. The next generation sensors move to digital binary readout and serve as the sensors of choice for an engineering prototype detector with limited coverage that will be deployed for the 2010 run at STAR. These digital readout generation sensors will contain on-chip correlated double sampling (CDS), fine grained threshold discrimination and a fast serial Low-Voltage Differential Signaling (LVDS) readout. 
A diagram showing the current development path and with the attendant evolution of the processing and readout requirements is shown in Figure 1.

\section{Data Processing in RDO and on chip by generation of sensor.}

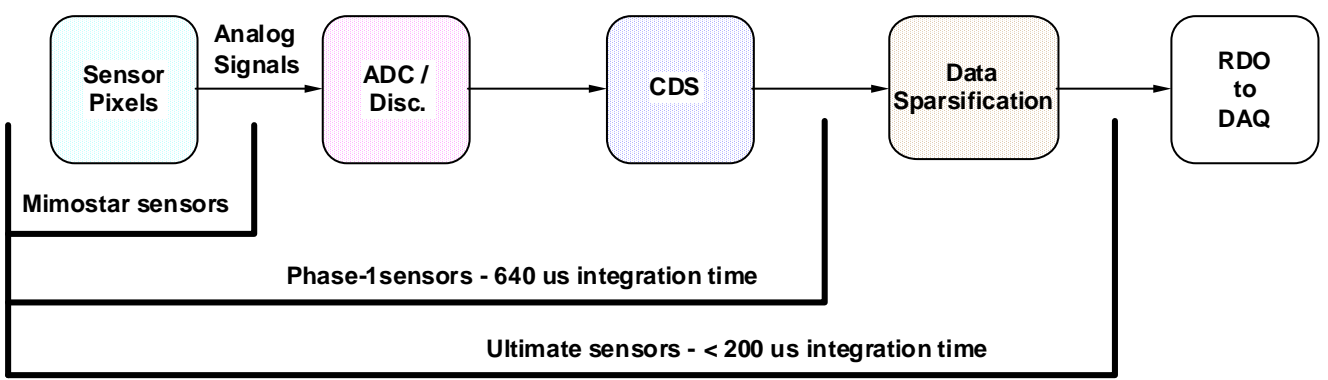

The RDO system design evolves with the sensor generation.

Figure 1. Diagram showing the sensor development path of sensors for the STAR Pixel detector at IPHC in Strasbourg, France. The readout data processing required is shown as a function of sensor generation. The first generation MimoSTAR sensors are read out via a rolling shutter type analog output. The next generation Phase-1 sensor integrates CDS and a column level discriminator to give a rolling shutter binary readout with a $640 \mu$ s integration time. The final generation Ultimate sensor integrates data sparsification and lowers the readout time to $<200 \mu \mathrm{s}$.

The MimoSTAR series of sensors are the current generation of sensors developed at IPHC. These are $50 \mathrm{MHz}$ multiplexed analog readout sensors with $30 \mu \mathrm{m} \times 30 \mu \mathrm{m}$ pixels in variously sized arrays. These MimoSTAR sensors have been tested, characterized and, with the exception of some yield issues, appear to be well understood. Some MimoSTAR series sensors test results are described later in this paper.

The next generation is named "Phase-1". This sensor will be based on the Mimosa- 8 and Mimosa-16 [4] sensors and will contain on-chip correlated double sampling and column level discriminators providing digital outputs in a rolling shutter configuration. The Phase- 1 will be a full sized $640 \times 640$ array resulting in a full $2 \mathrm{~cm} \times 2 \mathrm{~cm}$ sensor size. In order to achieve a 640 $\mu$ s integration time, the Phase- 1 sensor will be equipped with four LVDS outputs running at 160 MHz. The first delivery of wafers of this sensor design is expected in late 2008.

The final sensor is named "Ultimate". The Ultimate sensor contains all of the attributes of the Phase- 1 sensor with the pixel sub-arrays clocked faster to give a $<200 \mu$ s integration time and the integration of a run length encoding based data sparsification and zero suppression circuit. The reduction in data rate from the newly included data sparsification circuitry allows all of the data to be read out over one digital output per sensor within the integration time. The first prototypes of this design are expected to be delivered in the 2010 time frame.

\section{Conceptual Mechanical Designs and Prototyping}

The main requirement for the Pixel detector is to provide direct reconstruction of decays, including those of $\mathrm{D}^{0}$, that are displaced from the main vertex by approximately $100-150 \mu \mathrm{m}$. To provide this capability, the vertex detector has to be very thin to limit multiple Coulomb scattering (radiation length design limit of less than $0.5 \%$ per layer). This is planned to be 
achieved with sensors thinned down to a thickness of $50 \mu \mathrm{m}$, readout cables with aluminum conductors, extensive use of carbon fiber composite materials for the support structures, and air cooling of the system. In addition, spatial resolution of about $10 \mu \mathrm{m}$ is required with pixel positional stability in the detector array on the same scale. The sensor pixel pitch chosen for this application is $30 \mu \mathrm{m}$.

The final mechanical system is required to provide the stated angular coverage with appropriate overlaps in sensor areas to provide hermeticity and allow for detector alignment consistency checks. The present state of conceptual mechanical design for the Pixel detector currently contains two possible design candidates. One conceptual design is a concentric barrel arrangement consisting of 33 ladder assemblies. Each ladder is approximately $2 \mathrm{~cm}$ wide $\times 25$ $\mathrm{cm}$ long $\times 5 \mathrm{~mm}$ thick and consists of a rigid support assembly of reticulated vitreous carbon foam with a carbon fiber composite (CFC) skin, a read-out cable, and ten $2 \mathrm{~cm} \times 2 \mathrm{~cm}$ sensors. This full detector design consists of three sub-assemblies of rigid ladder elements. Each subassembly consists of three inner ladders and eight outer ladders joined with a CFC frame and subtending $1 / 3$ of the phi coverage. The detector is organized into effectively two sensor layers with 9 inner ladders and 24 staggered outer ladders resulting in a complete system that will contain approximately $135 \mathrm{M}$ pixels. This conceptual design of the Pixel detector is presented in Figure 2.

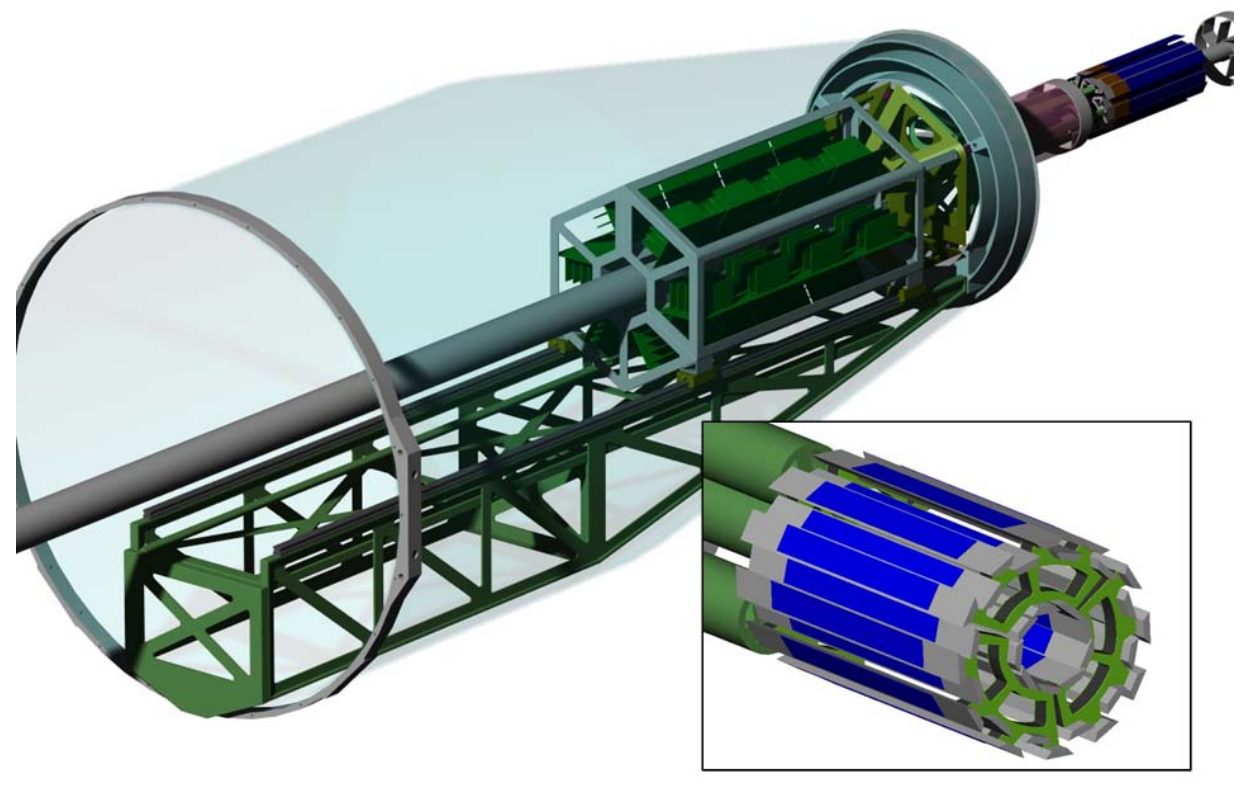

Figure 2. A conceptual design of the Pixel detector for the Heavy Flavor Tracker. The support structure with part of the readout electronics, presented in green, located inside the carbon fiber con structure, will provide capability for a quick replacement of the detector with a very high accuracy positioning (on the order of $20 \mu \mathrm{m}$ ). The inset presents a two layer barrel structure composed of 33 ladders each equipped with 10 MAPS sensors.

The inset of the figure shows the two layers of pixel sensors organized into two barrels at radii of 2.5 and $7 \mathrm{~cm}$ from the beam axis. The support structure represented in green is intended 
to provide the capability for quick detector replacement while preserving highly accurate detector positioning with the use of kinematic mounts.

Another conceptual design is an ALICE type design with a carbon fiber modified boxbeam support carriers. The box beam support carriers are composed of $250 \mu \mathrm{m} \mathrm{CFC}$ and support three outer detector rows and one inner detector row each. In this design, structural rigidity is provided by the carrier instead of each individual ladder. Rows of sensors mounted to a readout cable and thin support plate are fastened to and supported from the carrier mounting surfaces. A CAD model of this design is shown in Figure 3. In this conceptual design, the detector is organized into two half detector clamshell assemblies with five rigidly coupled carriers composing each clamshell half. The full detector then consists of ten box beam carriers and a total of 400 sensors. The radial spacing of layers is $2.5 \mathrm{~cm}$ and $8.0 \mathrm{~cm}$. In this configuration the detector would contain about $164 \mathrm{M}$ pixels on 40 ladders.

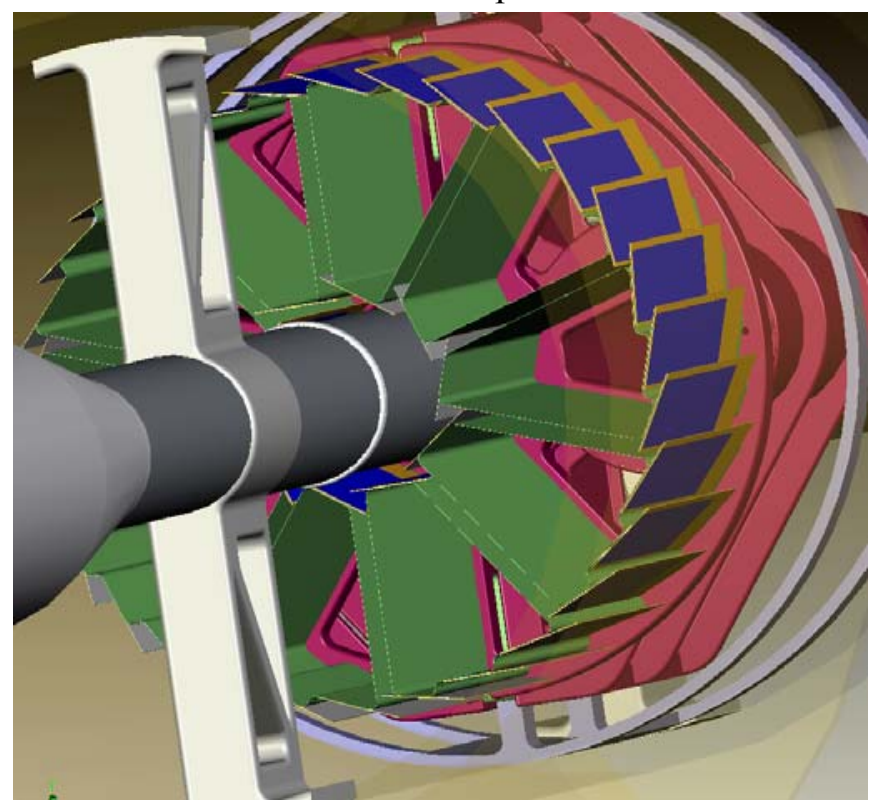

Figure 3. End view of an ALICE type modified box-beam carrier mechanical structure candidate currently undergoing FEA analysis. The kinematic mounting assemblies are shown in red.

Both designs are currently under FEA analysis for structural parameters and air cooling response. Each conceptual design has inherent strengths and weaknesses and complexity of manufacture will also be a strong component in the decision process. The design choices will be narrowed after full FEA analysis and when all mechanical criteria are met.

\section{Conceptual Readout Architecture}

The Pixel detector is an upgrade detector system proposed for the STAR experiment. It thus needs to integrate easily into the STAR environment. The requirements to integrate this detector system into STAR are;

- Triggered detector system fitting into existing STAR infrastructure and interfacing to the existing Trigger and DAQ systems. 
- Deliver full detector events to the STAR DAQ for event building at a rate equal to or greater than the TPC.

- Provide a total data rate to STAR at a manageable level (less than the TPC rate of approximately $1 \mathrm{MB} /$ event)

The data flow for a system based on analog output MimoSTAR series sensors consists of a synchronous parallel system of electronics for each sensor culminating in readout to DAQ at the ladder level. The analog data from each individual sensor is buffered and carried off of the ladder to readout electronics where is it digitized in a 12 bit ADC. The ADC sample is subtracted from the previous stored value for that pixel providing correlated double sampling, reducing the required resolution to 8 bits. This value is then fed into a high/low threshold discriminator and the results of that discriminator fed into a hit finder. The sensor serial data output is arranged as a repeating raster scan. This allows us to identify hits by doing threshold pattern recognition in a field programmable gate array (FPGA) over a synchronous window that sequentially examines a $3 \times 3$ array of pixels. When a hit is detected, only the center pixel address is read out. This algorithm is shown schematically in Figure 4.

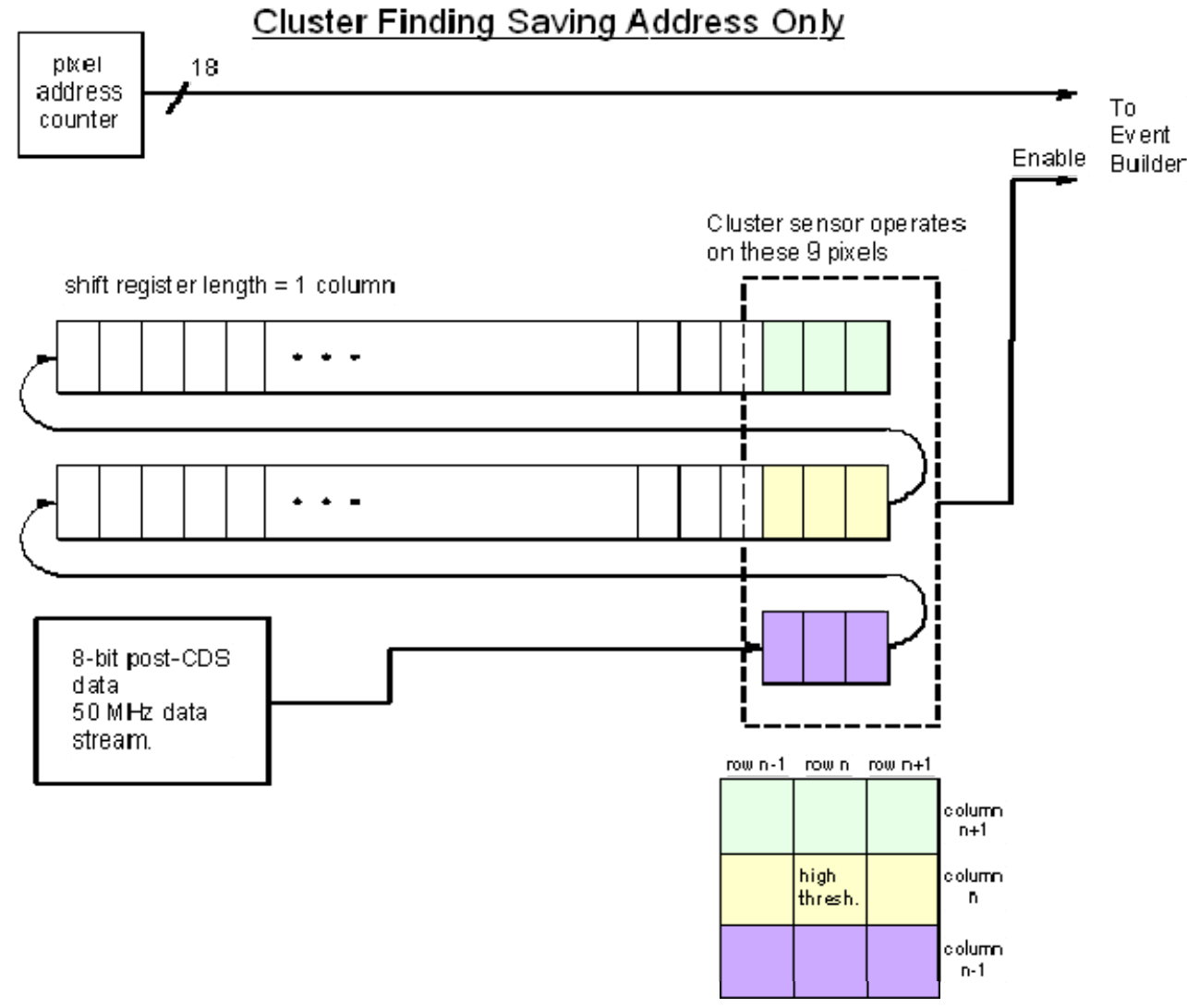

Figure 4. Schematic representation of the hit finder algorithm implemented in the prototype readout system for the Pixel detector. The sensor data is arranged as a raster scan clocked through a shift register. The algorithm allows performing zero suppression at the FPGA level. 12-bit ADC data from two full MimoSTAR-2 rows and additional 3 pixels from the next row are sent to a high/low threshold discriminator. At each clock cycle we examine the $3 \times 3$ pixel window highlighted in the schematic. The center pixel is compared to a high threshold and the surrounding 8 pixels with a lower threshold. If the 
center pixel and one of the 8 neighbors exceed their thresholds, the address of the central pixel is stored into a readout FIFO.

This hit finding algorithm provides the bulk of the data reduction required. Hit address data from each chip is read out into a multiple buffered system that then delivers events at the ladder level to the STAR DAQ system via the CERN developed ALICE DDL (Detector Data Link) [5] which consists of a PC based RORC (Read Out Receiver Card) linked via fiber optic cable to a detector based SIU (Source Interface Unit). We implement multiple simultaneous event buffering to allow us to take up to 1000 events per second despite having a $4 \mathrm{~ms}$ frame readout time. The hardware designed to accomplish this data flow is shown schematically (for a single ladder) in Figure 5.
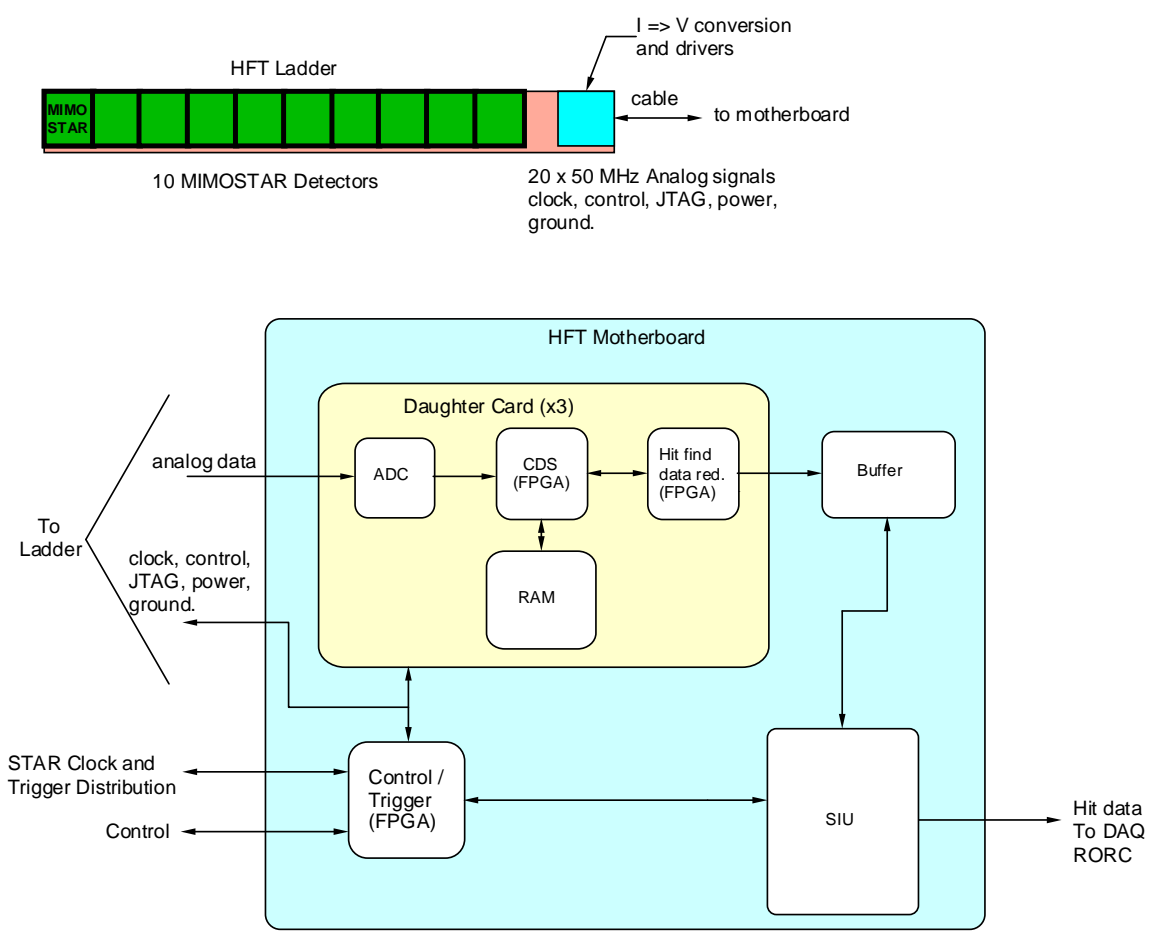

Figure 5. Schematic showing data flow for one ladder of the Pixel detector readout system. A Pixel MimoSTAR sensor based system would contain 33 parallel readout chains.

The raw data rate out of the ADCs at the front end is approximately $50.7 \mathrm{~GB} / \mathrm{s}$ for a full 33 ladder system. The detector operating parameters are listed below and allow for the following data rate calculations.

- $\quad$ Rate at R1 $(2.5 \mathrm{~cm})=52.9$ hits $/ \mathrm{cm}^{2}$

- Rate at R2 $(6.5 \mathrm{~cm})=10.75 \mathrm{hits} / \mathrm{cm}^{2}$

- Rate at R3 $(7.5 \mathrm{~cm})=8.76$ hits $/ \mathrm{cm}^{2}$

- 4 ms integration time

- 2.5 average hits / cluster

- Average trigger rate $=1 \mathrm{kHz}$

- $\quad$ Luminosity $=10^{27} \mathrm{~cm}^{-2} \mathrm{~s}^{-1}$ 
By implementing on-the-fly hit identification and reading out only the hit central address we reduce the data rate from $50.7 \mathrm{~GB} / \mathrm{s}$ to $114 \mathrm{MB} / \mathrm{s}$. This is shown schematically for the stages of the readout in Figure 6.

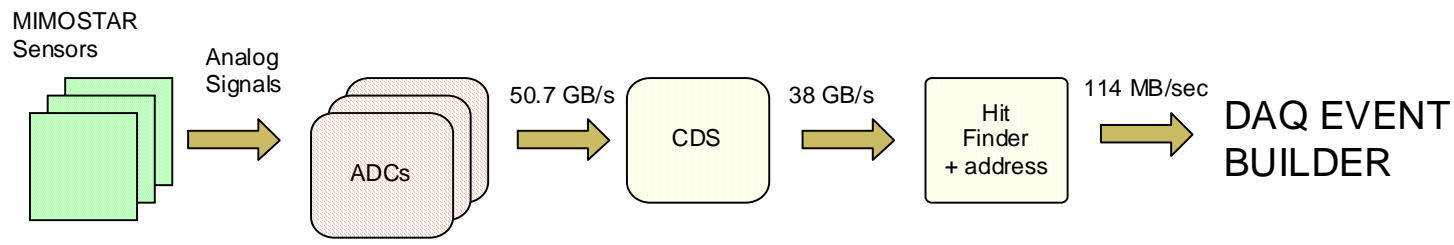

Figure 6. Data rates at the various stages of the MimoSTAR series sensor based readout system.

The described readout scheme reduces the raw data rate by a factor of approximately 1000 . This reduces the data rate of the HFT detector system to much less than that of the STAR TPC. The system is triggered via the current STAR triggering system and integrates into the STAR environment thus meeting the system requirements.

\section{MimoSTAR-2 Sensor based Telescope System Test}

As part of our incremental approach to solving the design challenges presented, we have been working with an existing prototype MimoSTAR-2 sensor. The MimoSTAR-2 is an analog output MAPS developed by our collaborators at IPHC with an array of $128 \times 128$ pixels. We have constructed a three sensors prototype detector in a telescope configuration. The telescope consists of three full die thickness sensors, each mounted to two-layer $25 \mu \mathrm{m}$ Kapton flex cables. The flex cables are glued to aluminum frames that define the telescope geometry with a planar spacing of $2.7 \mathrm{~mm}$. The sensors that comprise the telescope are then mounted into a plastic housing. This head assembly can be seen in Figure 7.

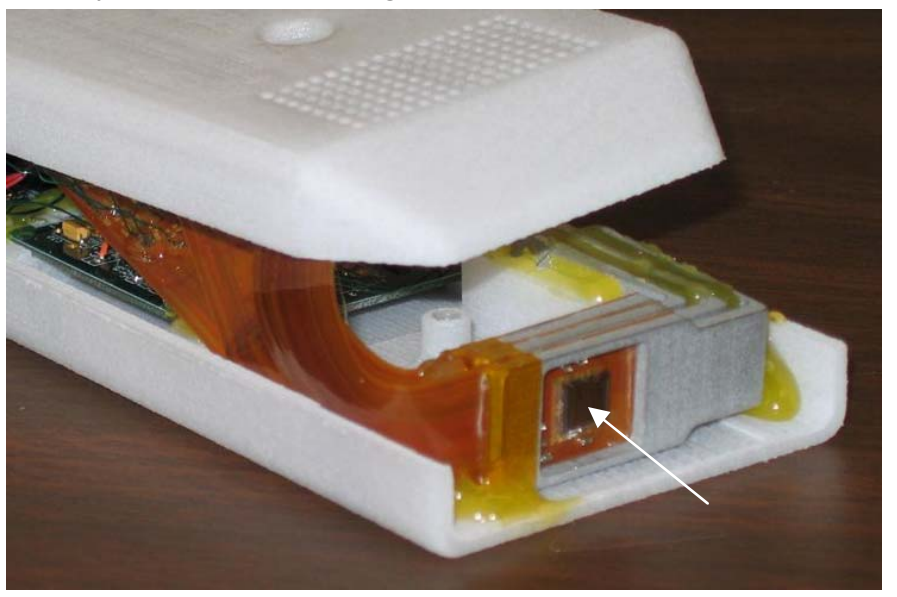

Figure 7. Picture of three sensor telescope constructed with three MimoSTAR-2 sensors. Kapton cables provide connection between sensors and control and readout electronics located on a readout PCB. The telescope was enclosed in a plastic housing head. Each sensor (indicated by the white arrow) is approximately $4 \mathrm{~mm} \times 4 \mathrm{~mm}$.

The prototype system is composed of four blocks as presented in Figure 8 . The sensor head contains the three MimoSTAR-2 sensors and the associated discrete component 
buffers/drivers. This head assembly is connected via individually shielded CAT-5 twisted pair cables to a motherboard, where the analog signals are routed to a daughter card to be digitized and processed. The daughter card performs CDS and data reduction through a real time cluster finding algorithm. The cluster address data is buffered, built into an event, and transferred to our data acquisition PC using a commercial Altera Stratix field programmable gate array (FPGA) development board (SDB) and a fiber optic connection. Latch-up protected power, clocks, resets and JTAG communication are provided to the sensor head from the motherboard / SDB combination. External triggers are accepted into the motherboard/daughter card. The system is run continuously and data flows through the digitization, CDS and cluster finding synchronously with a $50 \mathrm{MHz}$ clock. A fiber optic cable transmits the events, which are accumulated and built in response to a trigger, into the data acquisition PC (DAQ). This telescope readout system is a smaller and scalable version of the full detector readout system described in Section 4.

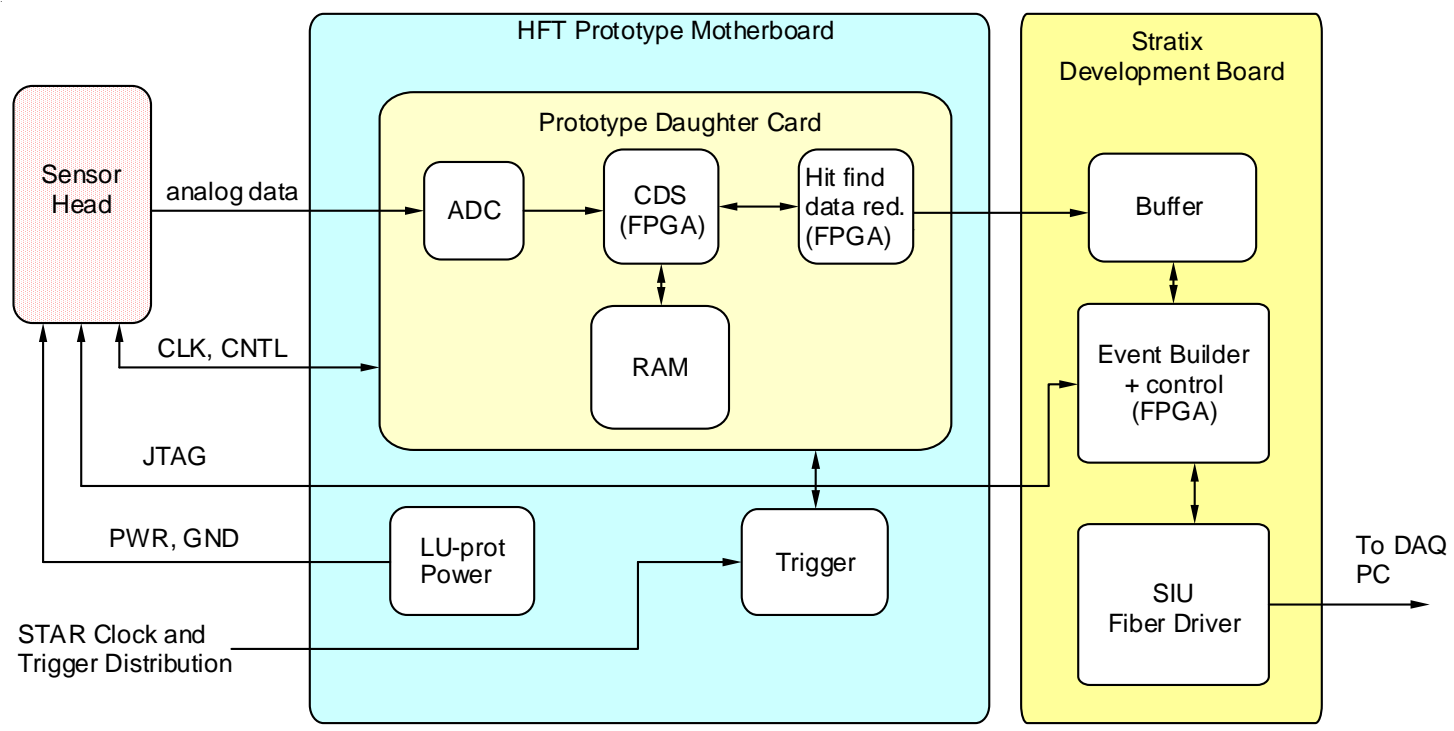

Figure 8. A schematic representation of the readout system.

The system described above was calibrated with an ${ }^{55} \mathrm{Fe}$ source and then tested with higher density hit rates (up to 200 clusters $/ \mathrm{cm}^{2}$ ) and further calibrated with $1.2 \mathrm{GeV}$ electrons at the Advanced Light Source (ALS) accelerator at LBNL. The fully tested and calibrated system was then mounted inside the TPC of the STAR detector for the last three weeks of the 2006-2007 run. The sensor head was placed near the interaction diamond with the sensor plane approximately perpendicular to the beam axis. The final location of the telescope head was about $5 \mathrm{~cm}$ below the beam pipe and about $145 \mathrm{~cm}$ from the center of the interaction region. The electronics box containing the prototype readout system was located inside the STAR magnet pole tip, at approximately the position expected to be used for the final Pixel detector electronics. The whole assembly operated in the nominal STAR magnetic field at $0.5 \mathrm{~T}$.

The goals of this test included measuring the performance of the telescope system in the STAR environment, checking for environmentally induced noise, measuring the charged particle density in STAR, and doing limited tracking with the sensor telescope. Integration of 
the telescope readout with the STAR control and trigger subsystems was also an important system level milestone.

Noise performance measured in the STAR environment was comparable to the results from laboratory and ALS tests. However, the overall noise performance of the telescope assembly might have masked some small environmentally induced noise increase. The charged particle density observed with the $1.7 \mathrm{~ms}$ integration time was about 3.9 clusters per sensor (about 25 clusters $/ \mathrm{cm}^{2}$ ). The average luminosity during the time the telescope was at STAR was $8 \times 10^{26} \mathrm{~cm}^{-2} \mathrm{~s}^{-1}$. The final Pixel detector at STAR is expected to operate at the RHIC II luminosity of approximately $8 \times 10^{27} \mathrm{~cm}^{-2} \mathrm{~s}^{-1}$ but with a much reduced integration time of 200 $\mu \mathrm{s}$. The expected hit densities in the final Pixel detector are 24 clusters $/ \mathrm{cm}^{2}$ on the outer sensors and 246 hits $/ \mathrm{cm}^{2}$ on the inner sensors. The MAPS pixel technology used in MimoSTAR sensors has proven to be compatible with these charged particle densities.

Data taken during the normal STAR operation included background tracks, the majority of which can be expected to originate form the beam-gas type interactions, and charged particle tracks originating at the collision point. A plot of the angular distribution of tracks registered by the telescope system is presented in Figure 9.

\section{track angle}

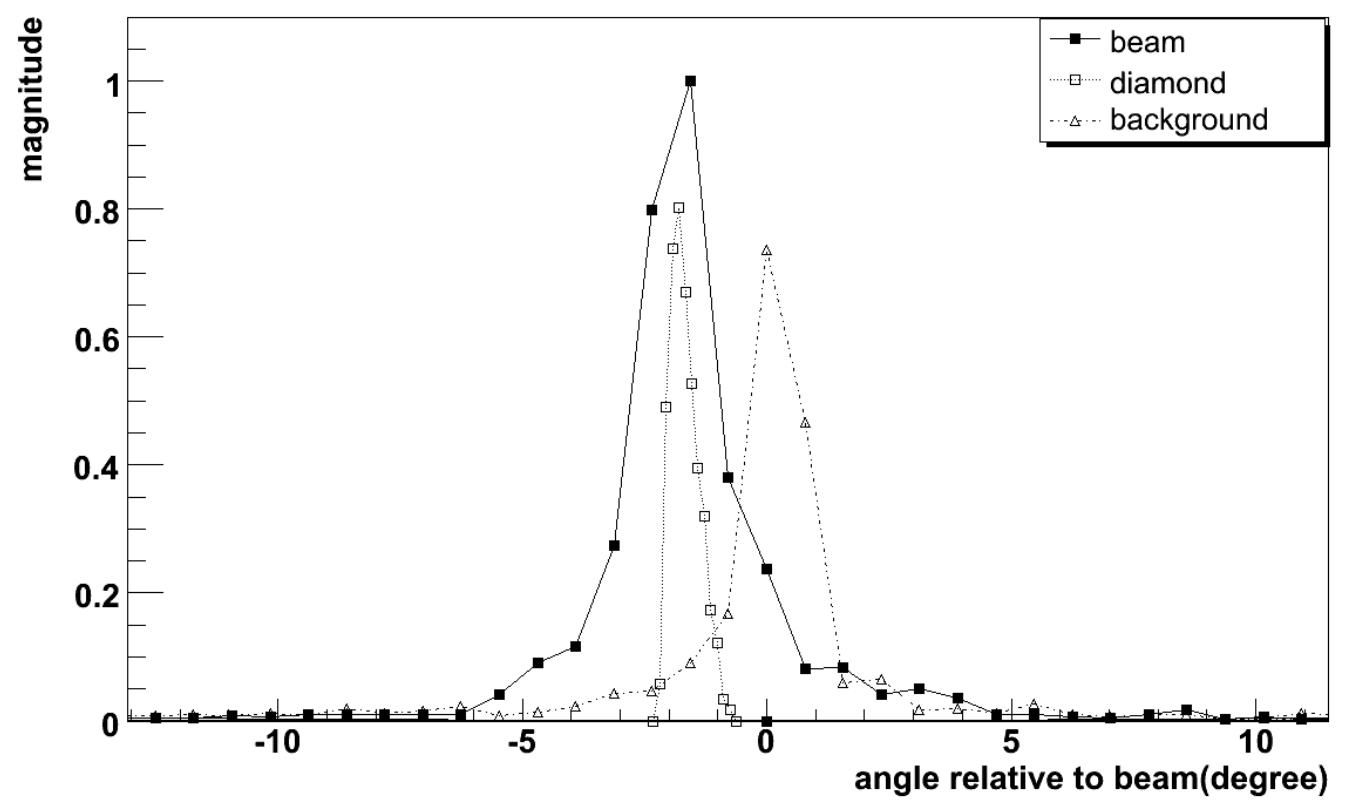

Figure 9. The angular distribution of tracks in the sensor head. The calculated distribution that corresponds to tracks from the interaction diamond (labeled "diamond) at STAR is shown as well as the measured angular distribution when the sensor was triggered by the STAR TPC trigger during normal beam interaction data taking (labeled "beam"). The background angular distribution (labeled "background") was taken during a special running mode where the RHIC beams were displaced such that there were no collisions in the interaction region and corresponds to beam-gas interactions and other backgrounds.

The angular distribution labeled "beam" was taken during normal data taking conditions at STAR and triggered by the STAR TPC trigger. The angular distribution labeled "diamond" is calculated from the known distribution of tracks in the STAR interaction diamond projected through our measured prototype detector position. The observed "beam" distribution peaks at 
the value calculated in the "diamond" but is wider and contains tails which is conjectured to be from scattering in the beam pipe. For a short run the particle beams at RHIC were displaced such that they did not collide in the STAR interaction region. The measured angular distribution of the background tracks (labeled "background") taken on a minimum bias trigger during this special running condition is also presented in Figure 9. This background distribution peaks at 0 degrees showing tracks parallel to the beam pipe as expected for beam-gas and other background sources. All curves in the plot have arbitrary scaling.

The prototype system was triggered from the STAR trigger system, all system power was controlled via the STAR slow control system, and events were read out via the DDL into a STAR standard receiver PC. The prototype telescope data was saved to the hard disk in the PC rather than being built into the STAR event structure.

\section{Conclusions}

The STAR Heavy Flavor Tracker upgrade group is working to extend the capabilities of the STAR detector in the heavy flavor domain by providing a tracking system that will allow for very high resolution vertex measurements. We have a well established sensor development plan in conjunction with IPHC that promises to deliver full reticule sized sensors with on sensor correlated double sampling, zero suppression and digital readout in the needed timeframes. In the mechanical support area we are pursuing a focused mechanical design process with two conceptual designs undergoing extensive development and FEA analysis. We have a well developed readout system design that meets the requirements for the detector upgrade system and reduces the raw data rate by a factor of nearly 1000. This readout architecture has been implemented in hardware, firmware and software and tested as the readout system for a prototype telescope composed of analog output MimoSTAR-2 sensor prototypes. A three element MimoSTAR-2 telescope has been constructed and coupled to the described RDO system with on-the-fly data sparsification. All aspects of the system performed as designed. The prototype MimoSTAR-2 telescope system performed measurements of the detector environment at STAR including induced electronic noise and the interfaces into the existing STAR infrastructure are now functional. We look forward to moving into system development with the next generation of digital output sensors.

\section{References}

[1] J. Adams, M.M. Aggarwal, Z. Ahammed, J. Amonett, B.D. Anderson, D. Arkhipkin, et al. Experimental and theoretical challenges in the search for the quark-gluon plasma: The STAR Collaboration's critical assessment of the evidence from RHIC collisions, Nuclear Physics A, Volume 757, Issues 1-2, First Three Years of Operation of RHIC, 8 August 2005, Pages 102-183.

[2] S. Kleinfelder, S. Li, F. Bieser, R. Gareus, L. Greiner, J. King, et al., A proposed STAR microvertex detector using Active Pixel Sensors with some relevant studies on APS performance, Nuclear Instruments and Methods in Physics Research Section A, Volume 565, Issue 1, Proceedings of the International Workshop on Semiconductor Pixel Detectors for Particles and Imaging - PIXEL 2005, 1 September 2006, Pages 132-138 
[3] Deptuch, G.; Berst, J.-D.; Claus, G.; Colledani, C.; Dulinski, W.; Gornushkin, Y.; et al. Design and testing of monolithic active pixel sensors for charged particle tracking, IEEE Transactions on Nuclear Science, Volume 49, Issue 2, Part 2, April 2002 Page(s):601 - 610.

[4] Y. Degerli, G.Deptuch, N. Fourches, A. Himmi, Y. Li, P. Lutz, F. Orsini, and M.Szelezniak, "A fast monolithic active pixel sensor with pixel-level reset noise suppression and binary outputs for charged particle detection ," IEEE, Trans. Nucl. Sci., vol. 52, no. 6, pp. 3186-3193, Dec. 2005.

[5] http://alice-proj-ddl.web.cern.ch/alice-proj-ddl/ 